\title{
Energetics of Coronal Mass Ejections
}

\author{
Prasad Subramanian $^{1}$ and Angelos Vourlidas ${ }^{2}$ \\ ${ }^{1}$ Inter-University Centre for Astronomy and Astrophysics, P.O Bag 4, Ganeshkhind, Pune - \\ 411007, India, email: psubrama@iucaa.ernet.in \\ ${ }^{2}$ Code 7663, Naval Research Lab, Washington, DC 20375, USA, email: vourlidas@nrl.navy.mil
}

\begin{abstract}
We examine the energetics of the best examples of flux-rope CMEs observed by LASCO in 1996-2001. We find that $69 \%$ of the CMEs in our sample experience a driving power in the LASCO field of view. For these CMEs which are driven, we examine if they might be deriving most of their driving energy by coupling to the solar wind. We do not find conclusive evidence to support this hypothesis. We adopt two different methods to estimate the energy that can possibly be released by the internal magnetic fields of the CMEs. We find that the internal magnetic fields are a viable source of driving power for these CMEs.
\end{abstract}

Keywords. Sun; corona, Sun: coronal mass ejections (CMEs), Sun: magnetic fields

\section{Introduction}

The energy budgets involved in Coronal mass ejection (CME) propagation at heights $\gtrsim 2 \mathrm{R}_{\odot}$ are indicative of the manner in which the CME interacts with streamers and the solar wind and also provide constraints on the energies required during the initiation phase. We study the evolution of potential and kinetic energies of 39 individual FR CMEs between 1997 and 2001. This comprises a complete sample of the best examples of FR CMEs observed by LASCO in 1996-2001 (out of about 4000 events). We first generate mass images of each CME and then calculate the potential and kinetic energies using a procedure similar to that described in Vourlidas et al. (2001). For 27/39 CMEs in our sample, the mechanical energy rises linearly with time, whereas 12/39 CMEs show no such trend. For the 27/39 CMEs for which mechanical energy rises linearly with time, there is a clear external driving power.

\section{Source of driving power: solar wind, or internal magnetic energy?}

If CMEs are propelled via coupling with the solar wind, larger CMEs should have larger driving powers, since they have larger areas for interacting with the solar wind and would therefore be better coupled with it. For the CMEs which have a driving power, figure 1 shows a scatterplot of size versus driving power. From figure 1, the correlation between the driving power and CME size is evidently poor, and we find little evidence to suggest that larger CMEs have more driving power. This casts doubt on the hypothesis that these CMEs are powered by momentum coupling with the ambient solar wind.

We have computed an estimate of the rate of energy released by the magnetic field advected by each CME using two different methods. We envisage that the propelling force is provided by some sort of $\vec{J} \times \vec{B}$ forces (due to misaligned magnetic fields and currents) within the flux rope. One method uses the average magnetic flux carried by near-earth magnetic clouds (e.g., Lepping et al. 1997) and assumes that this value is representative of the average magnetic flux that is frozen into a typical CME in our dataset. According to this method, the internal magnetic field of a CME can provide $0.744 \pm 1.352$ of 


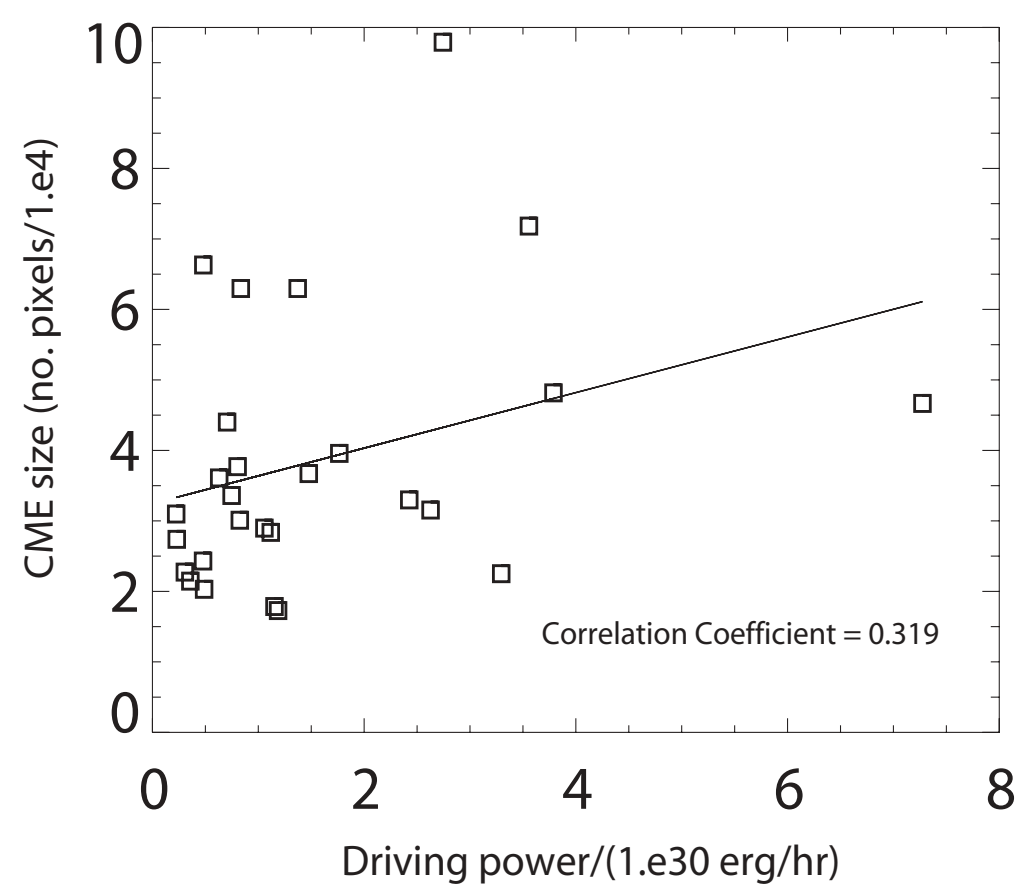

Figure 1. The mean size (in number of pixels) for CMEs plotted as a function of their driving power. The low correlation coefficient suggests that there is no evidence to claim that larger CMEs have larger driving powers.

the required driving power on the average. The other method uses direct estimates of the CME magnetic field from radio measurements (Bastian et al. 2001). According to this method, the internal magnetic field of a CME can provide $12.819 \pm 1.677$ of the required driving power on the average. The details of these calculations can be found in Subramanian \& Vourlidas (2004).

\section{Conclusions}

We find no evidence to suggest that the driven CMEs in our sample derive their driving power primarily via coupling with the solar wind. We employ two different approaches to investigate if release of the internal magnetic energy in the CME can possibly provide the driving power. One approach suggests that, on the average, energy released by the internal magnetic field of a CME can provide upto $74 \%$ of the required driving power. Another suggests that the energy released by the internal magnetic field of a CME can be around an order of magnitude greater than what is required to drive it.

\section{References}

Bastian, T.S., Pick, M., Kerdraon, A., Maia, D., \& Vourlidas, A. 2001, ApJ (Letters) 558, L65 Lepping, R.P., Szabo, A., DeForest, C.E., \& Thompson, B.J. 1997, in Proc. 31st ESLAB Symp., 'Correlated Phenomena at the Sun, in the Heliosphere and in Geospace, ESTEC, Noordwijk, The Netherlands, 22-25 September 1997 (ESA SP-415, December 1997)

Subramanian, P. \& Vourlidas, A. 2004, ApJ, submitted

Vourlidas, A., Subramanian, P., Dere, K.P., \& Howard, R.A. 2000, ApJ 534, 456 